TRANSACTIONS OF THE

AMERICAN MATHEMATICAL SOCIETY

Volume 173, November 1972

\title{
FUNCTIONS AND INTEGRALS
}

\author{
BY
}

\section{J. MALONE}

ABSTRACT. In $\$ 2$ a mapping of nonnegative functions is defined to be an integral if it has the following properties: $I(f) \geq 0, I(f)<\infty$ for some $f$, if $f \leq g$ then $I(f) \leq I(g), I(f+\rho)=2 I(f), I\left(\sum_{n=1}^{\infty} g_{n}\right) \leq \sum_{n=1}^{\infty} I\left(g_{n}\right)$. Given an integral $I$ a nonnegative function $f$ is defined to be a measurable function if $I(f+g)=I(f)+I(g)$ for all nonnegative functions $g$. If $f, g,\left(g_{n}\right)_{n=1}^{\infty}$ are measurable functions then the following functions are measurable: $f+g$, af for all $a \geq 0, \sum_{n=1}^{\infty} g_{n}, f-g$ if $f-g \geq 0$ and $I(g)<\infty$; also $\sum_{\eta=1}^{\infty} I\left(g_{n}\right)$ $=I\left(\sum_{n=1}^{\infty} g_{n}\right)$. An example shows if $f, g$ are measurable functions then

$\max \{f, g\}$ may fail to be a measurable function. If an integral has the property that if $f, g$ are measurable functions then $\max \{f, g\}$ is a ineasurable function, then the following functions are also measurable: $\min \{f, g\},|f-g|$, sup $g_{n}$ and under certain conditions $\lim _{n \rightarrow \infty}$ sup $g_{n}$, inf $g_{n}, \lim { }_{n \rightarrow \infty}$ inf $g_{n}$ whenever $\left(g_{n}\right)_{\eta=1}^{\infty}$ is a sequence of measurable functions. A theorem similar to Lebesgue $s$ dominated convergence theorem is shown to hold.

In $\$ 1$ the Lebesgue integral, which does not in general have the properties required to be an integral as defined in $\$ 2$, is used to obtain an integral $U$ which does. If $\mu$ is an outer measure and $\pi_{\mu}$ is the $\sigma$-algebra of $\mu$-measurable sets then the set of measurable functions defined in $\$ 2$ for the integral $U$ contains the usual set of $\pi_{\mu}$-measurable functions. $U$ has the property that if $f$ is a $\pi_{\mu}$-measurable function and if $\int_{X} f d \mu$ denotes the Lebesgue integral of $f$ on a set $X$ then $\int_{X} f d \mu=\mathbf{U}_{X} f d \mu$.

In $\oint_{3}$ it is shown that an, integral $I$ defined on a set $X$ induces an outer measure $\mu$. If $\mu$ is a regular outer measure, a representation theorem holds for $I$ : if $f$ is a nonnegative function and $U$ is the integral of $\$ 1$ then $I(f)$ $=\mathbf{U}_{X} f d \mu$. Regardless of whether or not the outer measure $\mu$ is regular a similar theorem can be obtained: if $f$ is a nonnegative $\pi_{\mu}$-measurable function then $I(f)=\mathbf{U}_{X} f d \mu$. The relationship between $\mu$-measurable sets and measurable functions is explored.

Wherever possible the definitions and notation have been taken from [2]. Throughout the following let $X$ be a set, let $N$ be the set of all positive integers, let $R$ be the set of all real numbers. Unless stated otherwise every function which appears is nonnegative; this is to be understood in the sense that if $f$ is defined on $X$ then $0 \leq f(x) \leq \infty$ for all $x \in X$.

1. The integral $U$. Let $X$ be a set $w$ ith an outer measure $\mu$ defined on it.

Received by the editors September 28, 1971.

AMS (MOS) subject classifications (1970). Primary 28A10, 28A20, 28A25, 28A30, 28-00.

Key words and phrases. Measurable functions, nonmeasurable functions, integrals of functions, countably subadditive norms, interchanging integrals and summations, interchanging integrals and limits, dominated convergence, outer measures, regular outer measures, Lebesgue integral, measurable sets, nonmeasurable sets, representation of an integral. 
Let $\prod_{\mu}$ be the $\sigma$-algebra of $\mu$-measurable subsets of $X$. For a nonnegative function $f$ defined on $X$ let $\int_{X} f d \mu$ be the Lebesgue integral of $f$.

This section will use the Lebesgue integral to define an 'upper' integral $\mathbf{U}$ with the properties:

$$
\int_{X} f d \mu=\mathbf{U}_{X} f d \mu
$$

whenever $f$ is an $\mathbb{M}_{\mu}$-measurable function,

$$
0 \leq \mathbf{U}_{X} f+g d \mu \leq \mathbf{U}_{X} f d \mu+\mathbf{U}_{X} g d \mu
$$

whenever $f, g$ are nonnegative functions. The purpose of defining and studying $\mathbf{U}$ is to give an example of a type of integral which will be examined in $\$ 2$.

Before starting the main development of this section, the construction of two sets $C, D$ which are not Lebesgue measurable will be sketched; $C, D$ will be such that $C \cap D=\varnothing$ and $0<\lambda(C)=\lambda(D)=\lambda(C \cup D)$ where $\lambda$ is Lebesgue measure. Let $\pi_{\lambda}$ be the set of Lebesgue measurable subsets of $R$. Let $M$ be the 'everywhere' nonmeasurable subset of $R$ which appears in Theorem (16.E) of [1]. A useful property of the set $M$ is that for any $B \in \mathbb{M}_{\lambda}$ it is true that $\lambda(B \cap M)=$ $\lambda\left(B \cap M^{\prime}\right)=\lambda(B)$. For purposes here take $B=[0,1] \subset R$ and let $C=B \cap M$, $D=B \cap M^{\prime}$ so that $C \cup D=B$ and $\lambda(C)=\lambda(D)=\lambda(C \cup D)=1$.

The useful feature of $C, D$ is that

$$
\lambda(C)+\lambda(D)=2 \lambda(C \cup D)
$$

In this sense $C, D$ are some 'worst possible' nonmeasurable sets. They will be used below to illustrate the behavior of various integrals.

Attention will now be turned to the central idea of this section: the development of an upper integral $\mathbf{U}$ such that

$$
0 \leq \mathbf{U}_{X} f+g d \mu \leq \mathbf{U}_{X} f d \mu+\mathbf{U}_{X} g d \mu
$$

whenever $f, g$ are nonnegative. That the Lebesgue integral does not have this property can be seen by taking $C, D$ as above and setting

Now

$$
\begin{array}{ll}
\xi_{C}(x)=1 & \text { if } x \in C, \\
\xi_{C}(x)=0 & \text { if } x \in R \cap C^{\prime}, \\
\xi_{D}(x)=1 & \text { if } x \in D, \\
\xi_{D}(x)=0 & \text { if } x \in R \cap D^{\prime} .
\end{array}
$$

$$
\begin{array}{ll}
\xi_{C}(x)+\xi_{D}(x)=1 & \text { if } x \in C \cup D, \\
\xi_{C}(x)+\xi_{D}(x)=0 & \text { if } x \in R \cap(C \cup D)^{\prime} .
\end{array}
$$

Hence $\int_{R} \xi_{C}+\xi_{D} d \lambda=\lambda(C \cup D)$. However $\int_{R} \xi_{C} d \lambda=0$ since 


$$
\inf \left\{\xi_{C}(x): x \in A, A \in \mathbb{M}_{\lambda}, \lambda(A)>0\right\}=0
$$

and

$$
\begin{aligned}
\int_{R} \xi_{C} d \lambda=\sup & \left\{\sum_{k=1}^{n} \inf \left\{\xi_{C}(x): x \in A_{k}\right\} \lambda\left(A_{k}\right):\right. \\
& \left.\left\{A_{1}, \cdots, A_{n}\right\} \text { is a measurable dissection of } R\right\} .
\end{aligned}
$$

Since each $A_{k} \in \mathbb{M}_{\lambda}$ it is true that

$$
\int_{R} \xi_{C} d \lambda=\sup \{0\}=0
$$

Since $\xi_{D}$ is similar it is true that

$$
0=\int_{R} \xi_{C} d \lambda+\int_{R} \xi_{D} d \lambda<\int_{R} \xi_{C}+\xi_{D} d \lambda
$$

Since the Lebesgue integral does not work it is necessary to find one that will. The first idea that presents itself might be to define for a nonnegative $f$

$$
\begin{aligned}
\mathbf{U}_{X} f d \mu=\inf & \left\{\sum_{k=1}^{n} \sup \left\{f(x): x \in A_{k}\right\} \mu\left(A_{k}\right):\right. \\
& \left.\left\{A_{1}, \cdots, A_{n}\right\} \text { is a measurable dissection of } X\right\} .
\end{aligned}
$$

This appears to work as long as $f$ is bounded $\mu$-a.e. and is positive only on a set of finite measure. Problems arise with functions like $x^{-1 / 2}$ on $[0,1]$ and $x^{-2}$ on $[1, \infty[$ for

$$
\int_{0}^{1} x^{-1 / 2} d \lambda=2 \text { but } \mathbf{U}_{0}^{1} x^{-1 / 2} d \lambda=\infty
$$

and

$$
\int_{1}^{\infty} x^{-2} d \lambda=1 \text { but } \mathbf{U}_{1}^{\infty} x^{-2} d \lambda=\infty
$$

One of the approaches which does appear to work for any function is to define $\mathbf{U}$ much as above but to go from measurable dissections with a finite number of $\mu$-measurable subsets of $X$ to 'countable dissections' $w$ ith a countable number of $\mu$-measurable pairwise disjoint subsets of $X$ such that $\bigcup_{k=1}^{\infty} A_{k}=X$. The approach here will be to use the Lebesgue integral and the properties of $\mathbb{M}_{\mu}$-measurable functions to define $\mathbf{U}$.

Hopefully by presenting things in terms of the Lebesgue integral the development will be more accessible to some readers than if $U$ were defined on countable dissections. In addition the details of showing that $\mathbf{U}$ does indeed treat $\mathbb{N}_{\mu}$-measurable functions just like the Lebesgue integral does can be omitted. Also, the approach presented here is suggestive of the treatment in $\$ 9$ of [2] of the integrals $I, \bar{I}, \bar{I}$ where $I$ looks not unlike the finite sums on measurable 
dissections (or integrals of simple functions), $\bar{I}$ looks not unlike the Lebesgue integral and $\bar{I}$ looks not unlike $\mathbf{U}$. However, the range of definition of $\mathbf{U}$ is rather more extensive than that of $\bar{I}$. In any case the different approaches give the same $\mathbf{U}$.

Definition 1.1. Let $f$ be any nonnegative function defined on a set $X$. Define

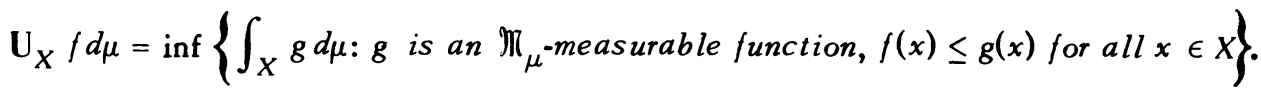

Theorem 1.2. Let $g$ be a nonnegative $\prod_{\mu}$-measurable function and let $f, b$ be nonnegative functions defined on a set $X . \mathrm{U}$ bas the following properties:

$$
\begin{aligned}
\mathbf{U}_{X} g d \mu & =\int_{X} g d \mu, \\
\mathbf{U}_{X} \alpha f d \mu & =\alpha \mathbf{U}_{X} f d \mu \quad \text { for } 0 \leq \alpha \leq \infty, \\
\mathbf{U}_{X} f+f d \mu & =\mathbf{U}_{X} f d \mu+\mathbf{U}_{X} f d \mu, \\
\mathbf{U}_{X} f+b d \mu & \leq \mathbf{U}_{X} f d \mu+\mathbf{U}_{X} b d \mu .
\end{aligned}
$$

Proof. (a), (b), (c) follow from the definition of $\mathbf{U}$; the reason for (c) as well as (b) will be seen in $\$ 2$. (d) can be obtained with the following: if $g_{f}, g_{b}$ are $\mathbb{M}_{\mu}$-measurable with $g_{f} \geq f, g_{b} \geq b$ then $g_{f}+g_{b}$ is $\mathbb{M}_{\mu}$-measurable and $g_{f}+g_{b}$ $\geq f+b$ when $f, b \geq 0$. Hence from the definition of $\mathbf{U}$ one sees that $\mathbf{U}_{X} f+b d \mu$ cannot be greater than $\mathbf{U}_{X} f d \mu+\mathbf{U}_{X} b d \mu$.

Using the functions $\xi_{C}, \xi_{D}$ which were defined before Definition 1.1 it is true that

$$
0<\mathbf{U}_{R} \xi_{C}+\xi_{D} d \lambda=\mathbf{U}_{R} \xi_{C} d \lambda=\mathbf{U}_{R} \xi_{D} d \lambda
$$

or

$$
\mathbf{U}_{R} \xi_{C}+\xi_{D} d \lambda<\mathbf{U}_{R} \xi_{C} d \lambda+\mathbf{U}_{R} \xi_{D} d \lambda
$$

and

$$
2 \mathbf{U}_{R} \xi_{C}+\xi_{D} d \lambda=\mathbf{U}_{R} \xi_{C} d \lambda+\mathbf{U}_{R} \xi_{D} d \lambda
$$

The next theorem will appear again in $\$ 2$. Here and elsewhere for the purpose of being specific whenever $f(x)=g(x)=\infty$ and $f-g$ appears set $f(x)-$ $g(x)=\infty$.

Theorem 1.3. Given $g \geq 0$ such that $\mathrm{U}_{X} g d \mu<\infty$ the following two statements are equivalent:

(a) $g$ is $\mathbb{M}_{\mu}$-measurable,

(b) $\mathbf{U}_{X} g+f d \mu=\mathbf{U}_{X} g d \mu+\mathbf{U}_{X} f d \mu$ for all $f \geq 0$. Also, (a) implies (b) even if $\mathbf{U}_{X} g d \mu=\infty$. 
Proof. First assume $g$ is $\prod_{\mu}$-measurable. Since

$$
\mathbf{U}_{X} f d \mu=\inf \left\{\int_{X} b d \mu: b \text { is } \pi_{\mu} \text {-measurable, } f \leq b\right\}
$$

there exists a sequence of functions $\left(b_{n}\right)_{n=1}^{\infty}$ such that $f \leq b_{n}$ for all $n \in N$ and

$$
\lim _{n \rightarrow \infty} \mathbf{U}_{X} b_{n} d \mu=\mathbf{U}_{X} f d \mu \text {. }
$$

Let $b=\inf b_{n}$. It is true that

(a) $b$ is $\mathbb{M}_{\mu}$-measurable,

(b) $f \leq b$,

(c) $\mathbf{U}_{X} b d \mu=\mathbf{U}_{X} f d \mu$.

It is true that

$$
\mathbf{U}_{X} b+g d \mu=\mathbf{U}_{X} b d \mu+\mathbf{U}_{X} g d \mu
$$

and

$$
\mathbf{U}_{X} f d \mu+\mathbf{U}_{X} g d \mu=\mathbf{U}_{X} b d \mu+\mathbf{U}_{X} g d \mu .
$$

The next step is to show that

$$
\mathbf{U}_{X} f+g d \mu=\mathbf{U}_{X} b+g d \mu .
$$

Only the case $\mathbf{U}_{X} f d \mu+\mathbf{U}_{X} g d \mu<\infty$ need be considered. Since $f \leq b$ it is true that

$$
f+g \leq b+g \text { and } \mathbf{U}_{X} f+g d \mu \leq \mathbf{U}_{X} b+g d \mu .
$$

If there existed $a>0$ such that

$$
\mathbf{U}_{X} f+g d \mu+\alpha=\mathbf{U}_{X} b+g d \mu,
$$

there would exist a function $e$ such that $e$ is $\mathbb{M}_{\mu}$-measurable, $e \geq f+g, \mathbf{U}_{X} e d \mu$ $<\mathbf{U}_{X} f+g d \mu+\alpha$. There would then exist $d$ such that $d$ is $\mathbb{M}_{\mu}$-measurable and $e=d+g$. Evidently $d \geq f$ and $\mathbf{U}_{X} d d \mu<\mathbf{U}_{X} b d \mu$. This would however contradict one of the properties of $b$. Hence it is true that

$$
\mathbf{U}_{X} f+g d \mu=\mathbf{U}_{X} b+g d \mu=\mathbf{U}_{X} b d \mu+\mathbf{U}_{X} g d \mu=\mathbf{U}_{X} f d \mu+\mathbf{U}_{X} g d \mu .
$$

This finishes the first part. For the remainder assume $\mathbf{U}_{X} g d \mu<\infty$. It still must be proven that if $g \geq 0$ has the property that

$$
\mathbf{U}_{X} f+g d \mu=\mathbf{U}_{X} f d \mu+\mathbf{U}_{X} g d \mu
$$

for all $f \geq 0$, then $g$ is $\prod_{\mu}$-measurable. This will be done by showing that if $g$ 
is not $\prod_{\mu}$-measurable, then there exists a function $f$ which turns out to also be not $\mathbb{M}_{\mu}$-measurable such that

$$
0<\mathbf{U}_{X} f+g d \mu<\mathbf{U}_{X} f d \mu+\mathbf{U}_{X} g d \mu .
$$

Let $g$ be a nonnegative function which is not $\prod_{\mu}$-neasurable. As was seen above there exists a function $b$ with the properties: $b$ is $\eta_{\mu}$-measurable, $g \leq b$, $\mathbf{U}_{X} g d \mu=\mathbf{U}_{X} b d \mu$. Now $b-g$ is a nonnegative function. It is also not $\prod_{\mu} \cdot$ measurable; were it $M_{\mu}$-measurable, then $b-(b-g)=g$ would be. Set $f=b-g$ so that $b=f+g$. It suffices to show $\mathbf{U}_{X} f d \mu>0$. Were it true that $\mathbf{U}_{X} f d \mu=0$, then for all $a>0$ with $\left.\left.B_{a}=f^{-1}(] a, \infty\right]\right)$ it would be true that $\mu\left(B_{a}\right)=0$ and hence $B_{a} \in M_{\mu}$. However, from the definition in $[2,11.2], f$ would then be $\mathbb{M}_{\mu}$-measurable. Since $f$ is not $\mathbb{M}_{\mu}$ measurable, $\mathbf{U}_{X} f d \mu>0$ and

$$
\mathbf{U}_{X} b d \mu=\mathbf{U}_{X} f+g d \mu<\mathbf{U}_{X} f d \mu+\mathbf{U}_{X} g d \mu .
$$

This theorem is also true for the Lebesgue integral. What can happen when $\mathbf{U}_{X} g d \mu=\infty$ can be seen in the function $b-\xi_{C}$ which appears in an example following Theorem 2.7.

One may have noted that the above treats only nonnegative functions. One reason can be found quickly enough. With sets $B, C$ from before Definition 1.1 let $\xi_{B}(x)=1$ if $x \in B, \xi_{3}(x)=0$ if $x \in R \cap B^{\prime}$ and similarly for $\xi_{C}$. If one writes for a nonnegative function $b, \mathbf{U}_{X}-b d \mu=-\mathbf{U}_{X} b d \mu$ it is true that $\mathbf{U}_{R} \xi_{B}-\xi_{C} d \lambda=\lambda\left(B \cap C^{\prime}\right)>0$ but $\mathbf{U}_{R} \xi_{B} d \lambda-\mathbf{U}_{R} \xi_{C} d \lambda=0$. Behavior of this kind shows the need to restrict Definition 1.1 to nonnegative functions. To treat functions generally it would be necessary to first split them into positive and negative parts and then treat each part as was done above. While such an approach is feasible and has been used in the development of the Lebesgue integral, here it would appear to compound the complexity which is already substantial while adding but little to whatever instructive value may be present.

In the following section attention is turned to a class of integrals which includes $\mathbf{U}$.

2. The integral $I$. This section will examine a particular collection of integrals. For each integral a set of measurable functions will be obtained and some of the properties of these measurable functions will be given.

Definition 2.1. Let $X$ be a set and let $f, g, g_{1}, g_{2}, g_{3}, \cdots$ be nonnegative functions defined on $X$. The real valued mapping $I$ will be called an integral when it has the properties

(a) $0 \leq I(f) \leq \infty$,

(b) there exists $f \geq 0$ such that $I(f)<\infty$,

(c) $I(f) \leq I(g)$ whenever $f \leq g$, 
(d) $I(f+f)=I(f)+I(f)$

(e) $I\left(\sum_{n=1}^{\infty} g_{n}\right) \leq \sum_{n=1}^{\infty} I\left(g_{n}\right)$.

The next four theorems show that the properties of Definition 2.1 are equivalent to a more extensive set of properties.

Theorem 2.2. Let $I$ be an integral and let 0 be the zero function: $0(x)=0$ for all $x \in X$. Then $I(0)=0$.

Proof. Using properties (b), (c), (d) $I(0)=I(0+0)=I(0)+I(0) \leq I(f)+I(f)$ $<\infty$. Hence $I(0)=0$.

Theorem 2.3. Let $I$ be an integral and let $f, g$ be nonnegative functions. Then $I(f+g) \leq I(f)+I(g)$.

Proof. Define $g_{3}=0, g_{4}=0, g_{5}=0, \ldots$. Then

$$
I(f+g)=I\left(f+g+\sum_{n=3}^{\infty} g_{n}\right) \leq I(f)+I(g)+\sum_{n=3}^{\infty} I(0)=I(f)+I(g) \text {. }
$$

Theorem 2.4. Let $I$ be an integral and let $f$ be a nonnegative function. If $0 \leq a \leq \infty$ then $I(\alpha f)=a I(f)$.

Proof. The theorem holds for $a=0,1,2$. If it is true for $a$ it is true for $2 \alpha$ and $\alpha / 2$ since

$$
I(2 \alpha f)=I(\alpha f)+I(\alpha f)=\alpha I(f)+\alpha I(f)=2 \alpha I(f)
$$

and

$$
\alpha I(f)=I(\alpha f)=I(\alpha / 2 f+\alpha / 2 f)=I(\alpha / 2 f)+I(\alpha / 2 f)
$$

or

$$
\alpha / 2 I(f)=I(\alpha / 2 f) .
$$

The theorem thus is true for $\alpha=2^{n}$ where $n$ is any integer. The theorem also holds when $\alpha=\sum_{k=1}^{j} 2^{n_{k}}$ where each $n_{k}$ is an integer. The case for $a=3$ will be done here; the others are similar. One wants to show $I(3 f)=3 I(f)$. From Theorem 2.3

$$
I(4 f) \leq I(3 f)+I(f) \leq 3 I(f)+I(f)
$$

but $I(4 f)=4 I(f)$ and hence $I(3 f)=3 I(f)$. Knowing the theorem holds whenever $\alpha$ can be written as a finite sum as was done above, one needs to show any nonnegative $a$ will work. This will be done here for $0<a<1$. The extension to any nonnegative $\alpha$ is straightforward. $\alpha$ can be written $\alpha=\sum_{k=1}^{\infty} 2^{-n_{k}}$ where each $n_{k}$ is a positive integer. It is true that

$$
\sum_{k=1}^{j} 2^{-n} k<a
$$


and

$$
I\left(\sum_{k=1}^{j} 2^{-n} k f\right)=\sum_{k=1}^{j} 2^{-n} k I(f)<I(\alpha f)
$$

however

Also

$$
I(\alpha f)=I\left(\sum_{k=1}^{\infty} 2^{-n} k f\right) \leq \sum_{k=1}^{\infty} 2^{-n} k I(f)=\alpha I(f)
$$

and

$$
\lim _{j \rightarrow \infty} I\left(\sum_{k=1}^{j} 2^{-n} k f\right) \leq I(\alpha f)
$$

but

$$
\lim _{j \rightarrow \infty} I\left(\sum_{k=1}^{j} 2^{-n} k f\right)=\sum_{k=1}^{\infty} 2^{-n} k I(f)
$$

$$
I(\alpha f)=\alpha I(f) .
$$

These theorems can be summarized in the following theorem.

Theorem 2.5. Let $I$ be an integral and let $f, g, g_{1}, g_{2}, g_{3}, \ldots$ be nonnegative functions defined on a set $X$. The list of properties in Definition 2.1 is equivalent to the following:
(a) $I(0)=0$,
(b) $0 \leq I(f) \leq \infty$,
(c) $I(f) \leq I(g)$ if $f \leq g$,
(d) $I(\alpha f)=\alpha I(f)$ if $0 \leq \alpha \leq \infty$,
(e) $I(f+g) \leq I(f)+I(g)$,
(f) $I\left(\sum_{n=1}^{\infty} g_{n}\right) \leq \sum_{n=1}^{\infty} I\left(g_{n}\right)$.

Evidently Theorem 2.5 could be used as the definition of an integral instead of Definition 2.1.

With the treatment given in $\$ 1$, the integral $U$ is an integral in the sense of Definition 2.1 while the Lebesgue integral is not.

The next step is to obtain a collection of measurable functions for a given integral $l$. This will be done in terms of the behavior of a function under integration and will make no reference to $\sigma$-algebras of sets. In addition no mention will be made of measures or outer measures. These entities will however appear in $\$ 3$.

Definition 2.6. Let $X$ be a set and let $I$ be an integral and let $g$ be any nonnegative function defined on $X, g$ is called a measurable function if it is true that $I(f+g)=I(f)+I(g)$ whenever $f$ is any nonnegative function defined on $X$. 
As was shown in Theorem 1.3 the set of functions which are measurable with respect to $U$ contains the set of $\prod_{\mu}$-measurable functions where $\mu$ is the outer measure used to define $U$.

It is true that any integral $I$ has a measurable function, the zero function, since $I(f+0)=I(f)+0$.

The uniform norm, $\|f\|_{u}[2,7.3]$, is defined $\|f\|_{u}=\sup \{|f(x)|: x \in X\}$. When restricted to nonnegative functions it is also an integral. It has only constant functions as measurable functions.

The $\mathscr{L}_{p}$-norm, $\|f\|_{p}[2,13.1]$, which for $1 \leq p<\infty$ and $f \geq 0$ is also an integral, is defined here $\|f\|_{p}=\left[\mathbf{U}_{X} f^{p} d \mu\right]^{1 / p}$. If $p>1$ and $X$ has more than one element with positive measure, then for every measurable $f$ it is true that $\|f\|_{p}$ $=0$. It might be anticipated that any treatment of measurable functions may be less than illuminating when applied to integrals for which the value of the integral of every measurable function is zero.

Next some properties of measurable functions are established.

Theorem 2.7. Let I be an integral and let $f, g, g_{1}, g_{2}, g_{3}, \ldots$ be nonnega. tive measurable functions. The following functions are measurable:

(a) $f+g$,

(b) $f-g$ if $g \leq f$ and $I(g)<\infty$,

(c) af if $0 \leq a \leq \infty$,

(d) $\sum_{n=1}^{\infty} g_{n}$.

It is also true that

(e) $I\left(\sum_{n=1}^{\infty} g_{n}\right)=\sum_{n=1}^{\infty} I\left(g_{n}\right)$.

Proof. Let $b$ be any nonnegative function. For (a) it is true that

$$
I(b+f+g)=I(b+f)+I(g)=I(b)+I(f)+I(g)=I(b)+I(f+g) .
$$

For (b) one needs to show that

$$
I(b+f-g)=I(b)+I(f-g) .
$$

Now

$$
I(b+g+f-g)=I(b)+I(g+f-g)=I(b)+I(g)+I(f-g)=I(b+g)+I(f-g),
$$

but

$$
I(b+g+f-g)=I(b+f-g)+I(g)
$$

and

$$
I(b+g)=I(b)+I(g)
$$

or 


$$
I(b+f-g)=I(b)+I(f-g) .
$$

For (c) one needs to show that

$$
I(b+a f)=I(b)+I(a f) .
$$

If $0<a<\infty, I(b / a+f)=I(b / a)+I(f)$ and $a I(b / a)=I(b), \alpha I(f)=I(a f)$. For (d) one needs to show that

Now

$$
I\left(b+\sum_{n=1}^{\infty} g_{n}\right)=I(b)+I\left(\sum_{n=1}^{\infty} g_{n}\right) .
$$

$$
I\left(b+\sum_{n=1}^{\infty} g_{n}\right) \leq I(b)+I\left(\sum_{n=1}^{\infty} g_{n}\right) \leq I(b)+\sum_{n=1}^{\infty} I\left(g_{n}\right) .
$$

For $k \in N$ using (a)

Now

$$
I\left(b+\sum_{n=1}^{k} g_{n}\right)=I(b)+I\left(\sum_{n=1}^{k} g_{n}\right)=I(b)+\sum_{n=1}^{k} I\left(g_{n}\right) .
$$

$$
\lim _{k \rightarrow \infty} \sum_{n=1}^{k} I\left(g_{n}\right)=\sum_{n=1}^{\infty} I\left(g_{n}\right)
$$

and

$$
I\left(b+\sum_{n=1}^{\infty} g_{n}\right)=I(b)+I\left(\sum_{n=1}^{\infty} g_{n}\right)=I(b)+\sum_{n=1}^{\infty} I\left(g_{n}\right) .
$$

For $b=0$ this gives $I\left(\sum_{n=1}^{\infty} g_{n}\right)=\sum_{n=1}^{\infty} l\left(g_{n}\right)$ which yields (e).

It can thus be seen that for an integral $I$ the set of measurable functions contains the zero function and is closed under countable addition, nonnegative multiplication and some subtractions. This set of functions can be obtained even though no mention is made of measures and measurable sets.

While there are cases for which (b) of Theorem 2.7 is true for $I(g)=\infty$, there are also cases that fail. The basic problem is that every function with an infinite integral is measurable. As long as subtractions are not involved this is of no importance. For an example of (b) which fails when $I(g)=\infty$ take the integral $\mathbf{U}$ of $\$ 1$ evaluated with Lebesgue measure and the set $C$ as before Definition 1.1. Define $b(x)=1$ for all $x \in R, b(x)-\xi_{C}(x)=1$ for all $x \in R \cap$ $C^{\prime}, b(x)-\xi_{C}(x)=0$ for all $x \in C$. It is true that $\mathbf{U}_{R} b d \lambda=\infty, \mathbf{U}_{R} b-\xi_{C} d \lambda=\infty$ and $b, b-\xi_{C}$ are measurable. However $b-\left(b-\xi_{C}\right)=\xi_{C}$ and $\xi_{C}$ is not measurable as was seen after. Theorem 1.2.

To this point for a given integral $I$ and its set of measurable functions nothing has been said about $\max \{f, g\}$ defined in $[2,7.1$. vii]. If $f, g$ are measurable functions is $\max \{f, g\}$ or $\min \{f, g\}$ a measurable function? For the following example the answer is no. 
Let $X$ be a set with elements $a, b, c$. Define for $a \geq 0$,

$$
\begin{array}{lll}
\alpha_{a}(a)=a, & a_{a}(b)=a / 2, & a_{a}(c)=0, \\
\alpha_{c}(a)=0, & a_{c}(b)=a / 2, & \alpha_{c}(c)=a .
\end{array}
$$

An integral $I$ will be defined for which $\alpha_{a}, \beta_{c}$ are the only measurable functions when $\alpha, \beta \geq 0$ and for which $\max \left\{\alpha_{a}, \beta_{c}\right\}$ is not measurable when $\alpha, \beta>0$. It will be useful however to first consider some of the properties of the functions $\alpha_{a}$, $\beta_{c}$.

(a) The family of functions $a_{a}+\beta_{c}$ for $\alpha, \beta \geq 0$ is closed under addition: for $\alpha, \beta, \gamma, \delta \geq 0$,

$$
\left(a_{a}+\beta_{c}\right)+\left(\gamma_{a}+\delta_{c}\right)=\alpha_{a}+\gamma_{a}+\beta_{c}+\delta_{c}=(\alpha+\gamma)_{a}+(\beta+\delta)_{c} .
$$

(b) If there are functions $f, g$ for which $f+g=\alpha_{a}+\beta_{c}$, it may not be true that either $f$ or $g$ has the form $\alpha_{a}^{\prime}+\beta_{c}^{\prime}$ with $\alpha^{\prime}, \beta^{\prime} \geq 0$. Define

$$
\begin{array}{lll}
f(a)=0, & f(b)=a^{\prime} / 2, & f(c)=0, \\
g(a)=a^{\prime}, & g(b)=0, & g(c)=0,
\end{array}
$$

for which $f+g=\alpha_{a}^{\prime}$. These functions will need to be considered when defining $I$ to insure

$$
I\left(\alpha_{a}+\beta_{c}\right)=I(f+g)<I(f)+I(g) .
$$

(c) Any function $f \geq 0$ can be written $f=a_{a}+\beta_{c}+z$ where $z$ has the form:

$$
z(a)=0, \quad z(b)=\gamma, \quad z(c)=0,
$$

for $\gamma>0$, in which case the expression for $f$ is unique

$$
z(a)=\gamma \geq 0, \quad z(b)=0, \quad z(c)=\gamma^{\prime} \geq 0,
$$

for $\gamma+\gamma^{\prime}>0$, in which case the restraint on $z$ is $z(a)+z(c)=\gamma+\gamma^{\prime}$. That $\mathrm{Z} 2$ does not yield a unique $z$ can be seen with the function $f(a)=2, f(b)=1$, $f(c)=2$, which can be written

$$
f=1_{a}+1_{c}+z \quad \text { with } z(a)=1, \quad z(b)=0, \quad z(c)=1
$$

or

$$
f=2_{a}+z \quad \text { with } z(a)=0, \quad z(b)=0, \quad z(c)=2,
$$

or in a number of other ways. Since each different $z$ will give a different pair $a_{a}, \beta_{c}$ the value of the integral of a function will have to be independent of how the function is written.

The integral $I$ is defined 


$$
\begin{aligned}
I\left(\alpha_{a}\right) & =\alpha_{a}(a)+\alpha_{a}(b)+\alpha_{a}(c)=\alpha+\alpha / 2+0=3 \alpha / 2 \geq 0, \\
I\left(\beta_{c}\right) & =\beta_{c}(a)+\beta_{c}(b)+\beta_{c}(c)=3 \beta / 2 \geq 0, \\
I\left(\alpha_{a}+\beta_{c}\right) & =I\left(\alpha_{a}\right)+I\left(\beta_{c}\right)=3 / 2(\alpha+\beta),
\end{aligned}
$$

so that $I\left(a_{a}+a_{c}\right)=3 a$. In attempting to make the functions $\alpha_{a}+\beta_{c}$ measurable and the functions $z$ not measurable, $I(z)$ will be set to the largest value allowed by (c) of Definition 2.1 which is

$$
I(z)=\inf \left\{I\left(\alpha_{a}+\beta_{c}\right): \alpha_{a}+\beta_{c}>z\right\} .
$$

For $z$ of the form Z1 with $z(b)=\gamma>0, z(a)=z(c)=0$, set $I(z)=3 \gamma$. For $z_{2}$ of the form Z2 with $z_{2}(a)+z_{2}(c)=\gamma>0, z_{2}(b)=0$, set $I\left(z_{2}\right)=3 \gamma / 2$. For $z(b)$ $=\gamma>0, z_{2}(a)+z_{2}(c)=2 \gamma$, one has

$$
3 \gamma=I\left(z+z_{2}\right)<I(z)+I\left(z_{2}\right)=6 \gamma,
$$

so that neither $z$ nor $z_{2}$ is measurable; hence no function of the form $\mathrm{Z1}$ or $\mathrm{Z2}$ is measurable.

For $f \geq 0$ of the form $f=\alpha_{a}+\beta_{c}+z, l$ is defined

$$
I(f)=I\left(\alpha_{a}+\beta_{c}\right)+I(z) .
$$

Using these relations one can show that $I$ is an integral and that a nonnegative function is measurable if and only if it has the form $a_{a}+\beta_{c}$.

The reason for constructing $I$ was to give an example of an integral for which $\max \{f, g\}$ is not measurable even though $f, g$ are measurable. Using $\alpha_{a}, \alpha_{c}$ let $m=\max \left\{a_{a}, \alpha_{c}\right\}$ for $\alpha>0$. Thus $m(a)=\alpha, m(b)=\alpha / 2, m(c)=\alpha$ and $m=a_{a}+z_{2}$ where $z_{2}(a)=z_{2}(b)=0, z_{2}(c)=a$. Hence $I(m)=3 a$. However $a_{a}+a_{c}-m=z$ where $z(b)=a / 2, z(a)=z(c)=0$. Thus

$$
\begin{aligned}
3 \alpha & =I\left(\alpha_{a}+\alpha_{c}\right)=I\left(\max \left\{\alpha_{a}, a_{c}\right\}+z\right) \\
& <I\left(\max \left\{\alpha_{a}, \alpha_{c}\right\}\right)+I(z)=3 \alpha+3 \alpha / 2 .
\end{aligned}
$$

Hence $\max \left\{a_{a}, \alpha_{c}\right\}$ is not measurable. Since $\min \left\{a_{a}, a_{c}\right\}=z$ it is not measurable either.

One might note that not all integrals share this property shown by the integral in the above example. For the integral $U$ of $\$ 1$ it is true that $\max \{f, g\}$ is measurable whenever $f, g$ are measurable. The following theorem expands on this.

Theorem 2.8. Let $I$ be an integral with the property that $\max \{f, g\}$ is measurable whenever $f, g$ are measurable. If $I(f+g)$ is finite then the following two functions are measurable:

(a) $\min \{f, g\}$, 
(b) $|f-g|$.

Let $\left(f_{n}\right)_{n=1}^{\infty}$ be a sequence of measurable functions,

(c) $\sup _{n} f_{n}$ is measurable.

If there exists $k \in N$ such that $I\left(\sup f_{k}, f_{k+1}, f_{k+2}, \ldots\right)$ is finite, then

(d) $\lim _{n \rightarrow \infty} \sup f_{n}$ is measurable.

If there exists $k \in N$ such that $I\left(f_{k}\right)$ is finite, then the following are measurable:

(e) $\inf _{n} f_{n}$,

(f) $\lim _{n \rightarrow \infty}$ inf $f_{n}$.

Proof. (a), (b) follow from Theorem 2.7 and the relations

$$
\begin{aligned}
\min \{f, g\} & =f+g-\max \{f, g\} \geq 0, \\
|f-g| & =2 \max \{f, g\}-(f+g) \geq 0 .
\end{aligned}
$$

For (c) write $g_{1}=f_{1}, g_{1}+g_{2}=\max \left\{f_{1}, f_{2}\right\}$. Now $g_{2}$ is measurable since $g_{2}$ $=\max \left\{f_{1}, f_{2}\right\}-f_{1} \geq 0$. Similarly write $\sum_{n=1}^{k} g_{n}=\max \left\{f_{1}, f_{2}, \ldots, f_{k}\right\}$ or

$$
g_{k}=\max \left\{f_{1}, f_{2}, \cdots, f_{k-1}, f_{k}\right\}-\max \left\{f_{1}, f_{2}, \cdots, f_{k-1}\right\} \geq 0 .
$$

Hence $\sup f_{n}=\sum_{k=1}^{\infty} g_{k}$ and $\sup f_{n}$ is the sum of a countable collection of measurable functions and is hence measurable.

For (d) let $s_{n}=\sup \left\{f_{n}, f_{n+1}, f_{n+2}, \ldots\right\}$ so that $\lim _{n \rightarrow \infty} s_{n}=$ $\lim _{n \rightarrow \infty} \sup f_{n}$. There exists $k \in N$ such that $I\left(s_{k}\right)$ is finite. For $n>k$, let $g_{n}=s_{k}-s_{n}$. Since each $g_{n}$ is measurable sup $g_{n}$ is measurable. Since $\lim _{n \rightarrow \infty} s_{n}=s_{k}-\sup g_{n}$ and $s_{k} \geq \sup g_{n}, \lim _{n \rightarrow \infty} s_{n}$ is measurable.

(e) can be established with arguments similar to those in (c), (d). (f) follows from (e), (c).

The necessity in (d) of Theorem 2.8 of requiring that $I\left(\sup \left\{f_{k}, f_{k+1}, \ldots\right\}\right)$ be finite for some $k$ is seen in an example. Given the set $C$ from before Definition 1.1 and the integral $U$ of $\$ 1$ defined with Lebesgue measure, define

$$
f_{n}(x)=1 \quad \text { if } x \in C, \quad f_{n}(x)=1 / n \quad \text { if } x \in R \cap C^{\prime} .
$$

It is true that $\lim _{n \rightarrow \infty} \sup f_{n}=\xi_{C}$ where $\xi_{C}(x)=1$ if $x \in C, \xi_{C}(x)=0$ if $x \in R \cap C^{\prime}$. It is true that $\mathbf{U}_{R} f_{n} d \lambda=\infty$ for all $n$ and that $\xi_{C}$ is not measurable as was shown after Theorem 1.2. Similar examples can be given for (e), (f) of Theorem 2.8.

Theorem 2.9. Let I be an integral and let $\left(f_{n}\right)_{n=1}^{\infty}$ be a sequence of nonnegative functions defined on a set $X$. Then

(a) $\sup _{n} I\left(f_{n}\right) \leq I\left(\sup _{n} f_{n}\right)$,

(b) I(inf $\left.{ }_{n} f_{n}\right) \leq \inf _{n} I\left(f_{n}\right)$.

The proof is omitted. 
Theorem 2.10. Let $I$ be an integral as in Theorem 2.8. If $\left(f_{n}\right)_{n=1}^{\infty}$ is a sequence of nonnegative measurable functions, then

(a) $I\left(\lim _{n \rightarrow \infty} \inf f_{n}\right) \leq \lim _{n \rightarrow \infty} \inf I\left(f_{n}\right)$.

For (b) and (c) let it be true that there exists $k \in N$ such that

$$
I\left(\sup \left\{f_{k}, f_{k+1}, f_{k+2}, \cdots\right\}\right)<\infty \text {. }
$$

Then

(b) $\lim _{n \rightarrow \infty} \sup I\left(f_{n}\right) \leq I\left(\lim _{n \rightarrow \infty} \sup f_{n}\right)$.

If $\lim _{n \rightarrow \infty}$ inf $f_{n}+g=\lim _{n \rightarrow \infty} \sup f_{n}$ where $I(g)=0$, then

(c) $\lim _{n \rightarrow \infty} I\left(f_{n}\right)$ exists and

$$
\lim _{n \rightarrow \infty} I\left(f_{n}\right)=I\left(\lim _{n \rightarrow \infty} \inf f_{n}\right)=I\left(\lim _{n \rightarrow \infty} \sup f_{n}\right)
$$

If $g=0, \lim _{n \rightarrow \infty} f_{n}=\lim _{n \rightarrow \infty} \inf f_{n}=\lim _{n \rightarrow \infty} \sup f_{n}$ and $\lim _{n \rightarrow \infty} I\left(f_{n}\right)=$ $I\left(\lim _{n \rightarrow \infty} f_{n}\right)$.

Proof. If there exists $k \in N$ such that for (a), $\inf \left\{I\left(f_{k}\right), I\left(f_{k+1}\right), \ldots\right\}=\infty$, the proof is straightforward. Otherwise there exists $k \in N$ such that $I\left(f_{k}\right)<\infty$ and $I\left(\inf f_{n}\right) \leq I\left(f_{k}\right)$. Let

$$
\inf \left\{f_{2}, f_{3}, \cdots\right\}=g_{1}+\inf \left\{f_{1}, f_{2}, \cdots\right\}=g_{1}+\inf _{n} f_{n} \text {. }
$$

Similarly for $k>2$ define

$$
\inf \left\{f_{k}, f_{k+1}, \cdots\right\}=\sum_{n=1}^{k-1} g_{n}+\inf _{n} f_{n}
$$

Each $g_{n}$ is measurable since for all $k \in N$ it is true that $\inf \left\{f_{k}, f_{k+1}, \ldots\right\}$ is measurable. Also

$$
\lim _{n \rightarrow \infty} \inf f_{n}=\sum_{n=1}^{\infty} g_{n}+\inf _{n} f_{n}
$$

For all $j \geq k>1$ it is true that

Hence

$$
I\left(\inf \left\{f_{k}, f_{k+1}, \cdots\right\}\right)=\sum_{n=1}^{k-1} I\left(g_{n}\right)+I\left(\inf _{n} f_{n}\right) \leq I\left(f_{j}\right)
$$

$$
I\left(\lim _{n \rightarrow \infty} \inf f_{n}\right)=\sum_{n=1}^{\infty} I(g)+I\left(\inf _{n} f_{n}\right) \leq \lim _{n \rightarrow \infty} \inf I\left(f_{n}\right) .
$$

For (b) there exists $k \in N$ such that $I\left(\sup \left\{f_{k}, f_{k+1}, \cdots\right\}\right)<\infty$. Let $\sup \left\{f_{k+1}, f_{k+2}, \cdots\right\}=\sup \left\{f_{k}, f_{k+1}, \cdots\right\}-g_{k}$. Similarly, for all $j>k$, let 


$$
\sup \left\{f_{j}, f_{j+1}, \cdots\right\}=\sup \left\{f_{k}, f_{k+1}, \cdots\right\}-\sum_{n=k}^{j-1} g_{n^{*}}
$$

Using the relations

and

$$
\lim _{n \rightarrow \infty} \sup _{n}=\sup \left\{f_{k}, f_{k+1}, \cdots\right\}-\sum_{n=k}^{\infty} g_{n}
$$

$$
I\left(\lim _{n \rightarrow \infty} \sup f_{n}\right)=I\left(\sup \left\{f_{k}, f_{k+1}, \cdots\right\}\right)-\sum_{n=k}^{\infty} I\left(g_{n}\right),
$$

the proof can be obtained in a manner similar to that in (a).

(c) follows fro:n

$$
\begin{aligned}
I\left(\lim _{n \rightarrow \infty} \sup f_{n}\right)= & I\left(\lim _{n \rightarrow \infty} \inf f_{n}\right) \leq \lim _{n \rightarrow \infty} \inf I\left(f_{n}\right) \leq \lim _{n \rightarrow \infty} I\left(f_{n}\right) \\
& \leq \lim _{n \rightarrow \infty} \sup I\left(f_{n}\right) \leq I\left(\lim _{n \rightarrow \infty} \sup f_{n}\right) .
\end{aligned}
$$

(c) of this theorem can be restated so that it resembles a form of Lebesgue's dominated convergence theorem [2, 12.24]:

If there exists some nonnegative function $s$ for which $I(s)$ is finite and $f_{n} \leq s$ for $n \in N$, and if $\lim _{n \rightarrow \infty} \inf f_{n}=\lim _{n \rightarrow \infty} \sup f_{n}$, then $\lim _{n \rightarrow \infty} f_{n}$ and $\lim _{n \rightarrow \infty} I\left(f_{n}\right)$ exist and $I\left(\lim _{n \rightarrow \infty} f_{n}\right)=\lim _{n \rightarrow \infty} I\left(f_{n}\right)$. One sees that $s \geq \sup f_{n}$, and that if $I(s)$ is finite then $I\left(\sup f_{n}\right)$ is also.

As has been shown $[2,12.25]$, (c) of this theorem may fail if one tries to extend it to the case where there is no dominating function or $I\left(\sup \left\{f_{k}, f_{k+1}, \ldots\right\}\right)$ $=\infty$ for all $k \in N$. It is possible however that

$$
I\left(\lim _{n \rightarrow \infty} \inf f_{n}\right)=\lim _{n \rightarrow \infty} I\left(f_{n}\right)=I\left(\lim _{n \rightarrow \infty} \sup f_{n}\right)<\infty,
$$

even though no dominating function exists. This can be seen in an example. Let the functions $f_{n k}$ be defined on $X=[0,1] \subset R$ with value zero except where defined otherwise. Define on the interval $\left.\left.\left.] 1 / 2^{1}, 1 / 2^{0}\right]=\right] 1 / 2,1\right]$ the function $f_{01}=2^{0}=1$ so that using the integral $\mathbf{U}$ of $\$ 1, \mathbf{U}_{X} f_{01} d \lambda=1 / 2$. Divide the interval $] 1 / 2^{2}, 1 / 2^{1}$ ] into two equal parts and define $f_{11}=2^{1}$ on ] $\left.1 / 2^{2}, 1 / 2^{2}+1 / 2^{3}\right]$ and $f_{12}=2^{1}$ on $\left.] 1 / 2^{2}+1 / 2^{3}, 1 / 2^{1}\right]$ so that $\mathbf{U}_{X} f_{11} d \lambda=$ $\mathbf{U}_{X} f_{12} d \lambda=2^{1} \cdot 1 / 2^{3}=1 / 2^{2}$. The interval $\left.] 1 / 2^{3}, 1 / 2^{2}\right]$ is divided into $2^{2}$ equal disjoint intervals of width $1 / 2^{3} \cdot 1 / 2^{2}=1 / 2^{5}$ and $f_{21}, f_{22}, f_{23}, f_{24}$ is each defined on one of these so that $f_{2 k}=2^{2}, \mathbf{U}_{X} f_{2 k} d \lambda=2^{2} \cdot 1 / 2^{5}=1 / 2^{3}$, $k=1,2,3,4$. The construction is continued by dividing the interval ] $1 / 2^{n+1}, 1 / 2^{n}$ into $2^{n}$ equal disjoint intervals of width $1 / 2^{n+1} \cdot 1 / 2^{n}=1 / 2^{2 n+1}$. The functions $f_{n k}, k=1,2, \ldots, 2^{n}$, are each defined on one of these intervals such that $f_{n k}=2^{n}$ and $\mathbf{U}_{X} f_{n k} d \lambda=2^{n} \cdot 1 / 2^{2 n+1}=1 / 2^{n+1}$. For this set of 
functions $\sup f_{n k} \geq 1 /(2 x)$ so that there is no dominating function; if $g \geq f_{n k}$ for all allowed $n, k$ then $g \geq 1 /(2 x)$ and $\mathbf{U}_{X} g d \lambda=\infty$. Nevertheless $\lim \inf f_{n k}=$ $\lim f_{n k}=\lim \sup f_{n k}$ and $\mathbf{U}_{X} \lim f_{n k} d \lambda=\lim \mathbf{U}_{X} f_{n k} d \lambda=0$.

It can be true that $\lim _{n \rightarrow \infty} I\left(f_{n}\right)$ exists and perhaps even equals $I\left(\lim _{n \rightarrow \infty} \inf f_{n}\right)$ or $I\left(\lim _{n \rightarrow \infty} \sup f_{n}\right)$ even though $I\left(\lim _{n \rightarrow \infty} \inf f_{n}\right) \neq$ $I\left(\lim _{n \rightarrow \infty} \sup f_{n}\right)$. Let $X=[0,1] \subset R$ and let $U$ be the integral of $\S 1$. Let the functions $\xi_{n k}$ be the characteristic functions of their respective intervals so that

$$
\begin{array}{llll}
\xi_{01}=1 & \text { on }[0,1] & \text { and } & \mathbf{U}_{X} \xi_{01} d \lambda=1, \\
\xi_{11}=1 & \text { on }[0,1 / 2] & \text { and } & \mathbf{U}_{X} \xi_{11} d \lambda=1 / 2, \\
\xi_{12}=1 & \text { on }[1 / 2,1] & \text { and } & \mathbf{U}_{X} \xi_{12} d \lambda=1 / 2, \\
\xi_{21}=1 & \text { on }[0,1 / 2] & \text { and } & \mathbf{U}_{X} \xi_{21} d \lambda=1 / 2^{2}
\end{array}
$$

so that $\xi_{n k}, k=1,2, \cdots, 2^{n}$, are defined to be the characteristic functions of the $2^{n}$ similar disjoint intervals of $\left.\left.[0,1]:\left[0,1 / 2^{n}\right],\right] 1 / 2^{n}, 2 / 2^{n}\right], \cdots$, ]$\left.\left(2^{n}-1\right) / 2^{n}, 1\right]$. Thus $\mathbf{U}_{X} \xi_{n k} d \lambda=1 / 2^{n}, k=1, \cdots, 2^{n}$. It is true that $\lim \sup \xi_{n k}=\xi_{01}=1$, $\lim \inf \xi_{n k}=0$, and $\lim \xi_{n k}$ does not exist. However $\lim \mathbf{U}_{X} \xi_{n k} d \lambda=0=\mathbf{U}_{X} \lim \inf \xi_{n k} d \lambda$ even though $\mathbf{U}_{X} \lim \sup \xi_{n k} d \lambda=1$.

This section closes with a consideration of the requirement in Theorem 2.10 that each function belonging to $\left(f_{n}\right)_{n=1}^{\infty}$ must be measurable. The reason for this can be roughly sketched. With $X=N$ let each $\xi_{n}$ be the characteristic function of the corresponding set $\xi_{1}(x)=1$ if $x \in\{1\}, \xi_{2}(x)=1$ if $x \in\{1,2\}, \ldots, \xi_{n}(x)$ $=1$ if $x \in\{1,2, \cdots, n\}, \cdots, \xi_{\infty}(x)=1$ if $x \in N$. For all $n \in N$ define $I\left(\xi_{n}\right)=1$ and let $I\left(\xi_{\infty}\right)=2$. It is possible to extend the definition of $I$ and make it an integral so that $I\left(\alpha \xi_{n}\right)=\alpha I\left(\xi_{n}\right)$ for $\alpha \geq 0, I\left(\xi_{k}+\xi_{n}\right) \leq I\left(\xi_{k}\right)+I\left(\xi_{n}\right)$, etc. Since $I\left(\xi_{\infty}\right)=2$ it can be shown that there is some $k \in N$ such that $\xi_{n}$ is not measurable if $n>k$. However, $\lim _{n \rightarrow \infty}$ inf $\xi_{n}=\xi_{\infty}$ so that $\lim _{n \rightarrow \infty}$ inf $I\left(\xi_{n}\right)=i<$ $I\left(\lim _{n \rightarrow \infty}\right.$ inf $\left.\xi_{n}\right)=2$ and in fact $I\left(\lim _{n \rightarrow \infty}\right.$ inf $\left.\xi_{n}\right)>\sup I\left(\xi_{n}\right)=1$.

3. A representation theorem for the integral $I$. The integral $I$ which appeared in $\$ 2$ will be used to induce an outer measure $\mu$ on the subsets of $X$. If $\mu$ is a regular outer measure it will be shown that

$$
I(f)=\mathbf{U}_{X} f d \mu,
$$

where $\mathbf{U}$ is the integral defined in $\$ 1$. If $f$ is a nonnegative $\pi_{\mu}$-measurable function this is equivalent to writing

$$
I(f)=\int_{X} f d \mu
$$

which says $I$ can be written as a Lebesgue integral.

All functions which appear are nonnegative. 
Definition 3.1. Let $X$ be a set and let $E$ be a subset of $X$. Define as in $[2,2.20]$,

$$
\xi_{E}(x)=1 \quad \text { if } x \in E, \quad \xi_{E}(x)=0 \quad \text { if } x \in X \cap E^{\prime} .
$$

$\xi_{E}$ is called the characteristic function of $E$. Define $\mu(E)=I\left(\xi_{E}\right)$.

The next theorem will show $\mu$ is an outer measure; $\mu$ will be referred to as the outer measure induced by $I$.

Theorem 3.2. $\mu$ defined in Theorem 3.1 is an outer measure as defined in $[2,10.2]$ :

(a) $0 \leq \mu(A) \leq \infty$ for all $A \subset X$,

(b) $\mu(\varnothing)=0$,

(c) $\mu(A) \leq \mu(B)$ if $A \subset B \subset X$,

(d) $\mu\left(\bigcup_{n=1}^{\infty} A_{n}\right) \leq \sum_{n=1}^{\infty} \mu\left(A_{n}\right)$ for all sequences $\left(A_{n}\right)_{n=1}^{\infty}$ of subsets of $X$.

Proof. See Theorem 2.5 and write

(a) $0 \leq I\left(\xi_{A}\right) \leq \infty$,

(b) $\xi_{\emptyset}=0$ and $I\left(\xi_{\emptyset}\right)=0$,

(c) $A \subset B$ implies $\xi_{A} \leq \xi_{B}$ and $I\left(\xi_{A}\right) \leq I\left(\xi_{B}\right)$,

(d) with $B=\bigcup_{n=1}^{\infty} A_{n}, I\left(\xi_{B}\right) \leq \sum_{n=1}^{\infty} I\left(\xi_{A_{n}}\right)$.

Associated with $\mu$ is the collection of $\mu$-measurable subsets of $X$.

Definition $[2,10.5]$. Let $X$ be a set and $\mu$ an outer measure on the power set of $X . A$ subset $A$ of $X$ is said to be $\mu$-measurable if

$$
\mu(T)=\mu(T \cap A)+\mu\left(T \cap A^{\prime}\right)
$$

for all $T \subset X$.

Associated with an integral $I$ is the collection of measurable nonnegative functions of Definition 2.6. In particular, there is the collection of measurable characteristic functions $\xi_{A}$ where $A \subset X$. The question arises: is there any relationship between $A \subset X$ being a $\mu$-measurable set and $\xi_{A}$ being a measurable function? The next theorem looks at one part of the problem.

Theorem 3.3. Let $\mu$ be the outer measure induced by the integral I. Let $A$ be a $\mu$-measurable set and $B$ a subset of $X$ sucb that $A \cap B=\varnothing$. Then

$$
I\left(\xi_{A}+\xi_{B}\right)=I\left(\xi_{A}\right)+I\left(\xi_{B}\right)
$$

Proof. $A$ is $\mu$-measurable and $\mu(A \cup B)=\mu((A \cup B) \cap A)+\mu\left((A \cup B) \cap A^{\prime}\right)$ $=\mu(A)+\mu(B)$. Hence $I\left(\xi_{A}+\xi_{B}\right)=I\left(\xi_{A}\right)+I\left(\xi_{B}\right)$.

For a given $\mu$-measurable set $A$ a limited collection of functions is treated by this theorem. It will be shown in Theorem 3.8 that if $\mu$ is a regular outer measure and if $A$ is $\mu$-measurable then $\xi_{A}$ is measurable. 
That Theorem 3.3 may fail if $A$ is not $\mu$-measurable can be seen in the example following Theorem 1.2.

Theorem 3.3 raises the question: if $\xi_{A}$ has the property that

$$
I\left(\xi_{A}+\xi_{B}\right)=I\left(\xi_{A}\right)+I\left(\xi_{B}\right) \text { whenever } A \cap B=\varnothing
$$

is $A \mu$-measurable? An example indicates not. Let $X$ be a set with disjoint subsets $A, B, C$. Let

$$
\begin{aligned}
I\left(\xi_{A}+\xi_{B}+\xi_{C}\right)=2, & I\left(\xi_{A}\right)=1, \quad I\left(\xi_{B}+\xi_{C}\right)=1, \\
I\left(\xi_{B}\right)=I\left(\xi_{C}\right)=1, & I\left(\xi_{A}+\xi_{B}\right)=I\left(\xi_{A}+\xi_{C}\right)=3 / 2 .
\end{aligned}
$$

$\xi_{B}+\xi_{C}$ is measurable since $I\left(\xi_{A}+\xi_{B}+\xi_{C}\right)=I\left(\xi_{A}\right)+I\left(\xi_{3}+\xi_{C}\right)$ and $A$ is the only nonvoid set not containing an element of $B \cup C$. $B \cup C$ is not $\mu$-measurable since

$$
\mu(A \cup \dot{B})<\mu((A \cup B) \cap(B \cup C))+\mu((A \cup B) \cap(B \cup C))=\mu(B)+\mu(A)
$$

or

$$
3 / 2=I\left(\xi_{A}+\xi_{B}\right)<I\left(\xi_{A}\right)+I\left(\xi_{B}\right)=1+1
$$

The situation here is that while $B \cup C$ is not $\mu$-measurable there is no set $D$ such that $(B \cup C) \cap . D=\varnothing$ and $\mu((B \cup C) \cup D)<\mu(B \cup C)+\mu(D)$. If $\mu$ is a regular outer measure such a $D$ can be found.

Definition [2, 10.40]. An outer measure $\mu$ on the power set of $X$ is said to be regular if for each $E \subset X$ there exists a $\mu$-measurable set $A \subset X$ such that $E \subset A$ and $\mu(A)=\mu(E)$.

Theorem 3.4. Let $\mu$ be a regular outer measure on a set $X$. If $E \subset X, \mu(E)$ $<\infty$, and $E$ is not $\mu$-measurable, then there exists $D \subset X$ such that $E \cap D=\varnothing$ and $\mu(E \cup D)<\mu(E)+\mu(D)$.

Proof. Since $\mu$ is a regular outer measure there exists a set $A$ such that $E \subset A, A$ is $\mu$-measurable and $\mu(E)=\mu(A)$. Since $E$ is not $\mu$-measurable $A \cap E^{\prime}$ is not $\mu$-measurable and $\mu\left(A \cap E^{\prime}\right)>0$. It is true that $E=A \cap E=A \cap\left(A \cap E^{\prime}\right)^{\prime}$ and $\mu(A)=\mu\left(E \cup\left(A \cap E^{\prime}\right)\right)<\mu(E)+\mu\left(A \cap E^{\prime}\right)=\mu(A)+\mu\left(A \cap E^{\prime}\right)$.

There exist outer measures induced by integrals which are not regular outer measures but which have in common with regular outer measures the property:

(3.5) If $A$ is not a $\mu$-measurable subset of $X$ and $\mu(A)<\infty$, then there exists a set $B \subset X$ such that $A \cap B=\varnothing$, and $\mu(A \cup B)<\mu(A)+\mu(B)$. Also $I\left(\xi_{A}+\xi_{B}\right)<I\left(\xi_{A}\right)+I\left(\xi_{B}\right)$ and $\xi_{A}$ is not a measurable function.

For an example of an outer measure $\mu$ which has property (3.5) but which is not a regular outer measure let $A, B$ be such that $A \cup B=X$ and $A \cap B=\varnothing$. Define $\left.I \xi_{A}+\xi_{B}\right)=1, I\left(\xi_{A}\right)=\lambda\left(\xi_{B}\right)=2 / 3$ so that $1=\mu(A \cup B)<\mu(A)+\mu(B)=4 / 3$. It is true that $A \cup B$ is $\mu$-measurable and is the only $\mu$-measurable set which contains either 
$A$ or $B$ both of which are not $\mu$-measurable. $\mu$ has property (3.5) even though it is not a regular outer measure. If $\mu$ were defined: $\mu(A \cup B)=1, \mu(A)=\mu(B)=1$ then $\mu$ would be a regular outer measure. There are examples of outer measures possessing property (3.5) which are not regular outer measures but which are not so simple as $\mu$. For those integral induced outer measures which have property (3.5), if $A$ is not $\mu$-measurable then $\xi_{A}$ is not measurable. Restating this: If $\xi_{A}$ is measurable then $A$ is $\mu$-measurable.

Before starting the development of the representation theorem it may be ap. propriate to include the following:

Theorem 3.6. Let $I$ be an integral which induces a regular outer measure $\mu$ on a set $X$. Let $\left(A_{n}\right)_{n=1}^{\infty}$ be a family of pairwise disjoint subsets of $X$ and for each $A_{n}$ let $B_{n}$ be a $\mu$-measurable set such that $A_{n} \subset B_{n} \subset X$ and $\mu\left(A_{n}\right)=\mu\left(B_{n}\right)$. Let $\left(\alpha_{n}\right)_{n=1}^{\infty}$ be a sequence of numbers such that $0 \leq \alpha_{n} \leq \infty$ for all $n \in N$. If $\left(B_{n}\right)_{n=1}^{\infty}$ is such that $\mu\left(B_{n} \cap B_{k}\right)=0$ if $n \neq k$, then for all $k \geq 1$,

$$
I\left(\sum_{n=1}^{k} \alpha_{n} \xi_{A_{n}}\right)=\sum_{n=1}^{k} \alpha_{n} I\left(\xi_{A_{n}}\right)=\sum_{n=1}^{k} \alpha_{n} I\left(\xi_{B_{n}}\right)=I\left(\sum_{n=1}^{k} \alpha_{n} \xi_{B_{n}}\right)
$$

and

$$
I\left(\sum_{n=1}^{\infty} a_{n} \xi_{A_{n}}\right)=\sum_{n=1}^{\infty} \alpha_{n} I\left(\xi_{A_{n}}\right)=\sum_{n=1}^{\infty} \alpha_{n} I\left(\xi_{B_{n}}\right)=I\left(\sum_{n=1}^{\infty} a_{n} \xi_{B_{n}}\right) .
$$

Proof. It is true that $I\left(\sum_{n=1}^{k} \xi_{3_{n}}\right)=\sum_{n=1}^{k} I\left(\xi_{B_{n}}\right)$ and $I\left(\sum_{n=1}^{\infty} \xi_{B_{n}}\right)=$ $\sum_{n=1}^{\infty} I\left(\xi_{B_{n}}\right)$. Also $I\left(\sum_{n=1}^{k} \xi_{A_{n}}\right)=I\left(\sum_{n=1}^{k} \xi_{B_{n}}\right)$ since for $k=2$ there is the function $\xi_{A_{1}}+\xi_{A_{2}}$ and the corresponding set $A_{1} \cup A_{2}$ for which there exists a $\mu$-measurable set $B$ such that $A_{1} \cup A_{2} \subset B$ and $\mu\left(A_{1} \cup A_{2}\right)=\mu(B)$. Were it true that $\mu(B)<\mu\left(B_{1}\right)+\mu\left(B_{2}\right)$ there would exist measurable sets $B \cap B_{1}$ and $B \cap B_{2}$ such that

$$
\mu\left(B \cap B_{1}\right)+\mu\left(B \cap B_{2}\right) \leq \mu(B)<\mu\left(A_{1}\right)+\mu\left(A_{2}\right) .
$$

Since $A_{1} \subset B \cap B_{1}$ and $A_{2} \subset B \cap B_{2}$ this cannot happen. Hence

$$
I\left(\xi_{A_{1}}+\xi_{A_{2}}\right)=I\left(\xi_{A_{1}}\right)+I\left(\xi_{A_{2}}\right)=I\left(\xi_{B_{1}}\right)+I\left(\xi_{B_{2}}\right)=I\left(\xi_{B_{1}}+\xi_{B_{2}}\right) .
$$

The proof for $k$ such that $3 \leq k<\infty$ is similar to $k=2$ and is omitted. It is possible to use the result for $3 \leq k<\infty$ to show that $I\left(\sum_{n=1}^{\infty} \xi_{A_{n}}\right)=\sum_{n=1}^{\infty} I\left(\xi_{A_{n}}\right)$ or $\mu\left(\cup_{n=1}^{\infty} A_{n}\right)=\sum_{n=1}^{\infty} \mu\left(A_{n}\right)=\sum_{n=1}^{\infty} \mu\left(B_{n}\right)$. It will next be shown that $I\left(\alpha_{1} \xi_{A_{1}}+\alpha_{2} \xi_{A_{2}}\right)=I\left(\alpha_{1} \xi_{A_{1}}\right)+I\left(\alpha_{2} \xi_{A_{2}}\right)$. For $\alpha_{1} \xi_{A_{1}}+\alpha_{2} \xi_{A_{2}}$ there is a corresponding function $\alpha_{2} \xi_{A_{1}}+\alpha_{1} \xi_{A_{2}}$; for this pair it is true that

$$
\begin{aligned}
& I\left(a_{1} \xi_{A_{1}}+a_{2} \xi_{A_{2}}+a_{2} \xi_{A_{1}}+a_{1} \xi_{A_{2}}\right) \\
& \quad=\left(a_{1}+\alpha_{2}\right) I\left(\xi_{A_{1}}+\xi_{A_{2}}\right)=\left(a_{1}+a_{2}\right)\left(I\left(\xi_{A_{1}}\right)+I\left(\xi_{A_{2}}\right)\right) .
\end{aligned}
$$


Also

$$
\begin{aligned}
& I\left(a_{1} \xi_{A_{1}}+a_{2} \xi_{A_{2}}+a_{2} \xi_{A_{1}}+a_{1} \xi_{A_{2}}\right) \\
& \quad \leq I\left(a_{1} \xi_{A_{1}}+a_{2} \xi_{A_{2}}\right)+I\left(a_{2} \xi_{A_{1}}+a_{1} \xi_{A_{2}}\right) \\
& \quad \leq a_{1} I\left(\xi_{A_{1}}\right)+a_{2} I\left(\xi_{A_{2}}\right)+a_{2} I\left(\xi_{A_{1}}\right)+a_{1} I\left(\xi_{A_{2}}\right) \\
& \quad=\left(a_{1}+a_{2}\right)\left(I\left(\xi_{A_{1}}\right)+I\left(\xi_{A_{2}}\right)\right) .
\end{aligned}
$$

Hence the two possible inequalities are actually equalities and $I\left(a_{1} \xi_{A_{1}}+a_{2} \xi_{A_{2}}\right)$ $=a_{1} I\left(\xi_{A_{1}}\right)+\alpha_{2} I\left(\xi_{A_{2}}\right)$. One might note that this was done without using the fact that $a_{1} \xi_{B_{1}}$ is a measurable function which is yet to be shown in Theorem 3.8. An argument of this type can be extended to show $I\left(\sum_{n=1}^{k} a_{n} \xi_{A_{n}}\right)=\sum_{n=1}^{k} \alpha_{n} I\left(\xi_{A_{n}}\right)$ $=\sum_{n=1}^{k} \alpha_{n} l\left(\xi_{B_{n}}\right)=I\left(\sum_{n=1}^{k} \alpha_{n} \xi_{B_{n}}\right)$. For the countable case it is true that $\sum_{n=1}^{k} \alpha_{n} I\left(\xi_{A_{n}}\right)=I\left(\sum_{n=1}^{k} \alpha_{n} \xi_{A_{n}}\right) \leq I\left(\sum_{n=1}^{\infty} \alpha_{n} \xi_{A_{n}}\right) \leq \sum_{n=1}^{\infty} \alpha_{n} I\left(\xi_{A_{n}}\right)$ and since

it is true that

$$
\lim _{k \rightarrow \infty} \sum_{n=1}^{k} \alpha_{n} I\left(\xi_{A_{n}}\right)=\sum_{n=1}^{\infty} a_{n} I\left(\xi_{A_{n}}\right)
$$

$$
I\left(\sum_{n=1}^{\infty} a_{n} \xi_{A_{n}}\right)=\sum_{n=1}^{\infty} a_{n} I\left(\xi_{A_{n}}\right)=\sum_{n=1}^{\infty} \alpha_{n} I\left(\xi_{B_{n}}\right)=I\left(\sum_{n=1}^{\infty} a_{n} \xi_{B_{n}}\right) .
$$

Theorem 3.7. Let $I$ be an integral which induces a regular outer measure $\mu$ on a set $X$. Let $f$ be a nonnegative function defined on $X$. Given $\epsilon>0$ there exists $\beta>0$ and sequences of subsets of $X,\left(C_{k}\right)_{k=-\infty}^{\infty},\left(D_{k}\right)_{k=-\infty}^{\infty}$, such that

(a) for all integer $k, D_{k}$ is $\mu$-measurable, $C_{k} \subset D_{k} \subset X, \mu\left(C_{k}\right)=\mu\left(D_{k}\right)$.

(b) For all $k \neq n, D_{k} \cap D_{n}=\varnothing, C_{k} \cap C_{n}=\varnothing$.

(c)

$$
\begin{aligned}
& \sum_{k=-\infty}^{\infty}(1+\beta)^{k} \xi_{C_{k}} \leq f \leq(1+\beta) \sum_{k=-\infty}^{\infty}(1+\beta)^{k} \xi_{D_{k}} \\
& \sum_{k=-\infty}^{\infty}(1+\beta)^{k} I\left(\xi_{C_{k}}\right)=I\left(\sum_{k=-\infty}^{\infty}(1+\beta)^{k} \xi_{C_{k}}\right) \\
& \leq I(f) \leq(1+\beta) I\left(\sum_{k=-\infty}^{\infty}(1+\beta)^{k} \xi_{D_{k}}\right) \\
& =(1+\beta) \sum_{k=-\infty}^{\infty}(1+\beta)^{k} I\left(\xi_{D_{k}}\right) \leq \sum_{k=-\infty}^{\infty}(1+\beta)^{k} I\left(\xi_{C_{k}}\right)+\epsilon .
\end{aligned}
$$

Proof. Assume $I(f)<\infty$; the other case is a straightforward extension of this one. The procedure will be to construct two functions $g, d$ such that $g \leq f \leq d$ and $I(g) \leq I(f) \leq I(d)$. It will also be true that $(1+\beta) I(g)=I(d)$ so that $I(d)-$ 
$I(f) \leq I(d)-I(g)=\beta I(g) \leq \beta I(f) . \quad \beta$ will then be chosen such that $\beta I(f)<\epsilon$.

Let $\beta$ be such that $\beta>0$. Define for all integer $k, A_{k}=\{x: x \in X, f(x) \geq$ $\left.(1+\beta)^{k}\right\}$ which can alternatively be written $A_{k}=f^{-1}\left(\left[(1+\beta)^{k}, \infty\right]\right)$. For each $A_{k}$ there exists $B_{k}$ such that $A_{k} \subset B_{k} \subset X, \mu\left(A_{k}\right)=\mu\left(B_{k}\right)<\infty$ and $B_{k}$ is $\mu$-measurable where $\mu$ is the regular outer measure induced by $I$. Define for each $B_{k}$, $D_{k}=B_{k} \cap\left(B_{k+1}\right)^{\prime}$. It is true that $\sup \left\{f(x): x \in D_{k}\right\} \leq(1+\beta)^{k+1}$ since if $f(x)$ $\geq(1+\beta)^{k+1}$, then $x \in A_{k+1}, x \in B_{k+1}, x \notin\left(B_{k+1}\right)^{\prime}, x \notin D_{k}$. It is also true that if $k \neq n$, then $D_{k} \cap D_{n}=\varnothing$. For all $k$ define $C_{k}=A_{k} \cap D_{k}$. It is true that $\mu\left(C_{k}\right)=\mu\left(D_{k}\right)$ since otherwise there would exist a $\mu$-measurable set $E$ such that $C_{k} \subset E \subset D_{k}$ and $\mu\left(C_{k}\right)=\mu(E)<\mu\left(D_{k}\right)$. Since $\mu\left(D_{k} \cap E^{\prime}\right)>0$ and $\left(D_{k} \cap E^{\prime}\right) \cap$ $A_{k}=\varnothing$ it would be true that $A_{k} \subset B_{k} \cap\left(D_{k} \cap E^{\prime}\right)^{\prime}$ and $\mu\left(A_{k}\right) \leq$ $\mu\left(B_{k} \cap\left(D_{k} \cap E^{\prime}\right)^{\prime}\right)<\mu\left(B_{k}\right)=\mu\left(A_{k}\right)$. Since this cannot happen it is true that $\mu\left(C_{k}\right)=\mu\left(D_{k}\right)$. Define $g=\Sigma_{k=-\infty}^{\infty}(1+\beta)^{k} \xi_{C_{k}}$. It is true that $g \leq f$. Also $I(g)=$ $\Sigma_{k=-\infty}^{\infty}(1+\beta)^{k} I\left(\xi_{C_{k}}\right)$ using Theorem 3.6. Let $D_{\infty}=\{x: x \in X, f(x)=\infty\}$. Since $I(f)<\infty$ it is true that $\mu\left(D_{\infty}\right)=0$. Define $d(x)=\infty$ if $x \in D_{\infty}$. Complete the definition of $d$ thus: $d=(1+\beta) \Sigma_{k=-\infty}^{\infty}(1+\beta)^{k} \xi_{D_{k}}$. It is true that $d \geq f$; indeed $d(x)>f(x)$ for all $x$ such that $0<f(x)<\infty$. It is also true that

$$
\begin{aligned}
I(d) & =(1+\beta) \sum_{k=-\infty}^{\infty}(1+\beta)^{k} I\left(\xi_{D_{k}}\right) \\
& =(1+\beta) \sum_{k=-\infty}^{\infty}(1+\beta)^{k} I\left(\xi_{C_{k}}\right)=(1+\beta) I(g) .
\end{aligned}
$$

Hence $g \leq f \leq d, I(g) \leq I(f) \leq I(d)$ and $\beta I(g) \geq \beta I(g)=I(d)-I(g) \geq I(d)-I(f)$. $\beta$ can be chosen such that $\beta I(f)<\epsilon$ and the theorem follows.

Theorem 3.8. Let $I$ be an integral which induces a regular outer measure $\mu$ on a set $X$. If $A$ is a $\mu$-measurable set then $\xi_{A}$ is a measurable function; which says given any nonnegative function $f$ defined on $X$ it is true that

$$
I\left(f+\xi_{A}\right)=I(f)+I\left(\xi_{A}\right) .
$$

Proof. Only the case for $I\left(\xi_{A}\right)<\infty$ need be considered. Let $f$ be such that $I(f)<\infty$. For $f$ and $\beta, \epsilon>0$ there exist families of sets $\left(C_{k}\right)_{k=-\infty}^{\infty},\left(D_{k}\right)_{k=-\infty}^{\infty}$ and the functions $g, d$ as defined in the proof of Theorem 3.7. Define $C_{-\infty}^{k=}=$ $D_{-\infty}=\{x: x \in X, d(x)=0\}$ and define for $-\infty \leq k \leq \infty, E_{k}=C_{k} \cap A, F_{k}=C_{k} \cap A^{\prime}$, $G_{k}=D_{k} \cap A$. It is true that $\bigcup_{k=-\infty}^{\infty} E_{k} \subset A, \bigcup_{k=-\infty}^{\infty} G_{k}=A$ and $\Sigma_{n=-\infty}^{\infty} I\left(\xi_{E_{k}}\right)$ $=\Sigma_{k=-\infty}^{\infty} I\left(\xi_{G_{k}}\right)=I\left(\xi_{A}\right)$. Also $I\left(\xi_{C_{k}}\right)=I\left(\xi_{E_{k}}+\xi_{F_{k}}\right)=I\left(\xi_{E_{k}}\right)+I\left(\xi_{F_{k}}\right)$. It is true that 


$$
\begin{aligned}
I\left(g+\xi_{A}\right) & =I\left(\sum_{k=-\infty}^{\infty}(1+\beta)^{k} \xi_{C_{k}}+\sum_{k=-\infty}^{\infty} \xi_{E_{k}}\right) \\
& =I\left(\sum_{k=-\infty}^{\infty}(1+\beta)^{k}\left(\xi_{E_{k}}+\xi_{F_{k}}\right)+\sum_{k=-\infty}^{\infty} \xi_{E_{k}}\right) \\
& =I\left(\sum_{k=-\infty}^{\infty}\left((1+\beta)^{k}+1\right) \xi_{E_{k}}+\sum_{k=-\infty}^{\infty}(1+\beta)^{k} \xi_{F_{k}}\right) \\
& =\sum_{k=-\infty}^{\infty}\left((1+\beta)^{k}+1\right) I\left(\xi_{E_{k}}\right)+\sum_{k=-\infty}^{\infty}(1+\beta)^{k} I\left(\xi_{F_{k}}\right) \\
& =\sum_{k=-\infty}^{\infty}(1+\beta)^{k}\left(I\left(\xi_{E_{k}}\right)+I\left(\xi_{F_{k}}\right)\right)+\sum_{k=-\infty}^{\infty} I\left(\xi_{E_{k}}\right) \\
& =I(g)+I\left(\xi_{A}\right) .
\end{aligned}
$$

It is true that $I(g)+I\left(\xi_{A}\right)=I\left(g+\xi_{A}\right) \leq I\left(f+\xi_{A}\right) \leq I(f)+I\left(\xi_{A}\right) \leq I(g)+\epsilon+I\left(\xi_{A}\right)$ and since given $\epsilon>0$ an expression of this form can be found it is true that $I\left(f+\xi_{A}\right)=I(f)+I\left(\xi_{A}\right)$ and $\xi_{A}$ is a measurable function.

Theorem 3.9. Let $I$ be an integral which induces a regular outer measure $\mu$ on a set $X$. Let $\left(A_{k}\right)_{k=1}^{\infty}$ be a family of $\mu$-measurable subsets of $X$. Let $\left(a_{k}\right)_{k=1}^{\infty}$ be such that $0 \leq a_{k} \leq \infty$ for all $k \in N$. The function

$$
d=\sum_{k=1}^{\infty} a_{k} \xi_{A_{k}}
$$

is a measurable function. Let $\left(d_{n}\right)_{n=1}^{\infty}$ be a sequence of measurable functions each of which is defined as $d$ is defined: each $d_{n}$ bas its own sequences $\left(A_{k}\right)_{k=1}^{\infty}$ and $\left(\alpha_{k}\right)_{k=1}^{\infty}$. Then the following functions are measurable:

$$
\inf _{n} d_{n}, \lim _{n \rightarrow \infty} \inf d_{n}, \sup _{n} d_{n}, \lim _{n \rightarrow \infty} \sup d_{n} .
$$

Proof. That $d$ is measurable is a restatement of Theorem 2.7. That $\sup d_{n}$ is measurable follows from Theorem 2.8 and the fact that if $A, B$ are $\mu$-measurable then $A \cup B$ is and $\xi_{A \cup B}=\max \left\{\xi_{A}, \xi_{B}\right\}$ Hence $\max \left\{\xi_{A}, \xi_{B}\right\}$ is measurable. If there exists a $d_{n}$ such that $I\left(d_{n}\right)$ is finite then inf $d_{n}$ is measurable. It is, however, also true that inf $d_{n}$ is measurable even if each $I\left(d_{n}\right)=\infty$. A proof of this will not be given; the argument is straightforward with the exception of one case that arises when for each $d_{n}$ there exists a set $A_{k}$ or sets for which $\mu\left(A_{k}\right)=\infty$. For a given nonnegative function $f$ such that $I(f)$ is finite and for $\beta>0$ there exists a sequence of subsets $\left(D_{i}\right)_{i=-\infty}^{\infty}$ as defined in Theorem 3.7. For each $A_{k}$ associated with $d_{n}$ the intersection of $A_{k}$ and each $D_{i}$ can be formed to give a sequence of subsets of $X$ each of which has finite measure. 
It can then be shown that $I\left(f+\inf d_{n}\right)=I(f)+I\left(\right.$ inf $\left.d_{n}\right)$. Once sup $d_{n}$ and inf $d_{n}$ are shown to be measurable $\lim _{n \rightarrow \infty} \inf d_{n}$ and $\lim _{n \rightarrow \infty} \sup d_{n}$ follow.

Next the representation theorem:

Theorem 3.10. Let $I$ be an integral which induces a regular outer measure $\mu$ on a set $X$. Let $f$ be any nonnegative function defined on $X$. Let $\mathrm{U}$ be the integral defined in (1.1). Then

$$
I(f)=\mathbf{U}_{X} f d \mu .
$$

Proof. For all $A \subset X$ it is true that $\mathbf{U}_{X} \xi_{A} d \mu=I\left(\xi_{A}\right)=\mu(A)$ so that $\mathbf{U}$ also induces a regular outer measure on $X$. For $\beta, d$ and a sequence of subsets $\left(D_{k}\right)_{k=-\infty}^{\infty}$ as in the proof of Theorem 3.7 it is true that $d=\Sigma_{k=-\infty}^{\infty}(1+\beta)^{k} \xi_{D_{k}}$ and

$$
I(d)=\sum_{k=-\infty}^{\infty}(1+\beta)^{k} I\left(\xi_{D}\right)=\sum_{k=-\infty}^{\infty}(1+\beta)^{k} \mathbf{U}_{X} \xi_{D} d \mu=\mathbf{U}_{X} d d \mu .
$$

For $f \geq 0$ and $I(f)<\infty$ it is true that $I(f)=\mathbf{U}_{X} f d \mu$. Assuming otherwise either $I(f)<\mathbf{U}_{X} f d \mu$ or $I(f)>\mathbf{U}_{X} f d \mu$. For the latter case there would exist a $d$ and $\left(D_{k}\right)_{k=-\infty}^{\infty}$ as in the proof of Theorem 3.7 such that

$$
I(f)>\mathbf{U}_{X} d d \mu=I(d) \geq \mathbf{U}_{X} f d \mu .
$$

However $d \geq f$ and it is true that $I(d) \geq I(f)$. The case for the assumption $I(f)<$ $\mathbf{U}_{X} f d \mu$ is similar. The proof for $I(f)=\infty$ is straightforward. Hence

$$
\mathbf{U}_{X} f d \mu=I(f) \text {. }
$$

Knowing an integral $I$ induces an outer measure $\mu$ a similar theorem can be obtained:

Theorem 3.11. Let $l$ be an integral which induces an outer measure $\mu$ on $a$ set $X$. Let $f$ be any nonnegative $\Pi_{\mu}$-measurable function defined on $X$. Let $\mathbf{U}$ be the integral defined in (1.1). Then

$$
I(f)=\mathbf{U}_{X} f d \mu .
$$

A proof which will not be given can be obtained which is similar to the proof of Theorem 3.10 and uses a theorem similar to Theorem 3.7 which is valid for $M_{\mu}$-measurable functions.

Theorem 3.11 also holds for the Lebesgue integral when it is used instead of the integral $\mathrm{U}$.

The rest of this section contains an assortment of topics that help round out the discussion. An integral $I$ induces an outer measure $\mu$ on a set $X$ for which there is a family of $\mu$-measurable sets. There is also a family of characteristic 
functions of the form $\xi_{A}$ which are measurable. The question arises: is there any relation between $A$ being $\mu$-measurable and $\xi_{A}$ being measurable? In the next two examples the answer is no. In the discussion above starting with Theorem 3.3 and ending just before Theorem 3.6 some aspects of this question were examined when the measurability of $\xi_{A}$ was determined by looking only at its behavior under integration with other 'disjoint' characteristic functions. Here the actual measurabllity of $\xi_{A}$ as defined in (2.6) will be examined.

It will first be shown that there exists a set $X$ and an integral $I$ such that with $B, C$ subsets of $X$ such that $B \cap C=\varnothing, \xi_{B \cup C}$ or equivalently $\xi_{B}+\xi_{C}$ is a measurable function even though $B \cup C$ is not ' $\mu$-measurable. Let $X$ be a set with disjoint single element subsets $A, B, C$. Extend the definition of $I$ which appeared in an example following Theorem 3.3 so that if $\alpha \geq 0$,

$$
\begin{aligned}
I\left(\alpha\left(\xi_{A}+\xi_{B}+\xi_{C}\right)\right) & =2 \alpha, \quad I\left(\alpha \xi_{A}\right)=\alpha, \\
I\left(\alpha\left(\xi_{B}+\xi_{C}\right)\right) & =\alpha, \quad I\left(\alpha \xi_{B}\right)=I\left(\alpha \xi_{C}\right)=\alpha, \\
I\left(\alpha\left(\xi_{A}+\xi_{B}\right)\right) & =I\left(\alpha\left(\xi_{A}+\xi_{C}\right)\right)=3 \alpha / 2 .
\end{aligned}
$$

Any function $f \geq 0$ defined on $X$ will have the form $f=a \xi_{A}+\beta_{B} \xi_{B}+\beta_{C} \xi_{C}$ with $\alpha_{s} \beta_{B}, \beta_{C} \geq 0$. Let $\beta=\min \left\{\beta_{B}, \beta_{C}\right\}$ and assume $\beta_{B} \geq \beta_{C}$ so $\beta_{C}=\beta$, $\beta_{B}=\beta+\beta_{1}$. Thus $f=\alpha \xi_{A}+\beta\left(\xi_{3}+\xi_{C}\right)+\beta_{1} \xi_{B}$. Deflne

$$
I\left(\alpha \xi_{A}+\beta\left(\xi_{B}+\xi_{C}\right)+\beta_{1} \xi_{B}\right)=I\left(\alpha \xi_{A}+\beta_{1} \xi_{B}\right)+\beta I\left(\xi_{B}+\xi_{C}\right)
$$

and define

$$
I\left(\alpha \xi_{A}+\beta_{1} \xi_{B}\right)=1 / 2\left(I\left(\alpha \xi_{A}\right)+I\left(\beta_{1} \xi_{B}\right)+\max \left\{I\left(\alpha \xi_{A}\right), I\left(\beta_{1} \xi_{B}\right)\right\}\right) .
$$

$\beta_{C} \geq \beta_{B}$ is treated similarly. It can be shown that $I$ is an integral and that $\xi_{B}+\xi_{C}$ is measurable even though $B \cup C$ is not $\mu$-measurable:

$$
\mu(A \cup B)<\mu((A \cup B) \cap(B \cup C))+\mu\left((A \cup B) \cap(B \cup C)^{\prime}\right)=\mu(B)+\mu(A) .
$$

Hence if $\xi_{A}$ is measurable then $A$ need not be $\mu$-measurable.

An integral can be defined which has the opposite behavior: $A$ is $\mu$-measurable and $\xi_{A}$ is not measurable. Let $X$ be the union of the pairwise disjoint single element sets $A, B, C$. For $0 \leq \alpha<\infty$, define $I\left(\alpha \xi_{A}\right)=\alpha$; for $0 \leq \beta \leq 1$, define $I\left(\xi_{B}+\beta \xi_{C}\right)=I\left(\beta \xi_{B}+\xi_{C}\right)=1+\beta / 2$ so that $I\left(\xi_{B}\right)=I\left(\xi_{C}\right)=1$, $I\left(\xi_{B}+\xi_{C}\right)=3 / 2 ;$ for $0<\alpha<\infty, 0 \leq \beta \leq 1$, define

$$
I\left(\alpha \xi_{A}+\xi_{B}+\beta \xi_{C}\right)=I\left(\alpha \xi_{A}+\beta \xi_{B}+\xi_{C}\right)=\max \{\alpha+1+\beta(1+\beta) / 4,1+\beta / 2\}
$$

so that $I\left(\xi_{A}+\xi_{B}\right)=I\left(\xi_{A}\right)+I\left(\xi_{B}\right)=2=I\left(\xi_{A}+\xi_{C}\right)$ and $I\left(\xi_{A}+\xi_{B}+\xi_{C}\right)=I\left(\xi_{A}\right)$ $+I\left(\xi_{B}+\xi_{C}\right)$. Hence $A$ is a measurable set. For $0 \leq \alpha<\infty, 0 \leq \beta \leq 1,0 \leq \gamma$ $<\infty$, define 


$$
I\left(\gamma\left(\alpha \xi_{A}+\xi_{B}+\beta \xi_{C}\right)\right)=\gamma I\left(\alpha \xi_{A}+\xi_{n}+\beta \xi_{C}\right)
$$

and

$$
I\left(\gamma\left(\alpha \xi_{A}+\beta \xi_{B}+\xi_{C}\right)\right)=\gamma I\left(\alpha \xi_{A}+\beta \xi_{B}+\xi_{C}\right) .
$$

It can be shown $I$ is an integral. $\xi_{A}$ however is not a measurable function since

$$
I\left(\xi_{A}\right)+I\left(\xi_{B}+\xi_{C} / 2\right)=1+1+1 / 4=2+1 / 4
$$

and

$$
I\left(\xi_{A}+\xi_{B}+\xi_{C} / 2\right)=1+1+(1 / 2)(1+1 / 2) / 4=2+3 / 16
$$

Hence if $A$ is $\mu$-measurable then $\xi_{A}$ need not be measurable.

Theorems 3.4 and 3.8, however, show that for a regular outer measure $\mu$ the two statements are equivalent if $\mu(A)<\infty$ :

(a) $A$ is $\mu$-measurable,

(b) $\xi_{A}$ is measurable.

The two preceding examples show that there exist integrals for which there is no logical relation between these two statements. Are these two statements equivalent only for regular outer measures? An example indicates not.

Let $X$ be the union of the disjoint single element sets $A, B, C$. Define for $\alpha \geq 0$ :

$$
\begin{aligned}
I\left(\alpha \xi_{A}\right) & =\alpha, \quad I\left(\alpha \xi_{B}\right)=I\left(\alpha \xi_{C}\right)=3 \alpha / 4, \\
I\left(\alpha \xi_{A}+\alpha \xi_{B}\right) & =I\left(\alpha \xi_{A}\right)+I\left(\alpha \xi_{B}\right)=7 \alpha / 4 \\
I\left(\alpha \xi_{A}+\alpha \xi_{C}\right) & =I\left(\alpha \xi_{A}\right)+I\left(\alpha \xi_{C}\right)=7 \alpha / 4 \\
I\left(\alpha \xi_{B}+\alpha \xi_{C}\right) & =\alpha<I\left(\alpha \xi_{B}\right)+I\left(\alpha \xi_{C}\right)=6 \alpha / 4 \\
I\left(\alpha \xi_{A}+\alpha \xi_{B}+\alpha \xi_{C}\right) & =I\left(\alpha \xi_{A}\right)+I\left(\alpha \xi_{B}+\alpha \xi_{C}\right)=2 \alpha .
\end{aligned}
$$

$B$ is not $\mu$-measurable since $1=\mu(B \cup C)<\mu(B)+\mu(C)=6 / 4$. $\xi_{B}$ is not measurable since $1=I\left(\xi_{B}+\xi_{C}\right)<I\left(\xi_{B}\right)+I\left(\xi_{C}\right)=6 / 4$. $C, \xi_{C}$ are similar. $B, C$ are the only subsets of $X$ which are not $\mu$-measurable. Hence if $D \subset X$ is not $\mu$ measurable then $\xi_{D}$ is not measurable; if $\xi_{D}$ is measurable then $D$ is $\mu$-measurable. The sets $A, B \cup C$ are $\mu$-measurable and $I$ will be extended so that $\xi_{A}, \xi_{B}+\xi_{C}$ are measurable. For $\alpha, \beta, \gamma \geq 0$, define $I\left(\alpha \xi_{A}+\beta \xi_{B}+\gamma \xi_{C}\right)=$ $I\left(\alpha \xi_{A}\right)+I\left(\beta \xi_{B}+\gamma \xi_{C}\right)$. For $\beta \geq \gamma$ define

$$
I\left(\beta \xi_{B}+\gamma \xi_{C}\right)=I\left(\gamma\left(\xi_{B}+\xi_{C}\right)+(\beta-\gamma) \xi_{E}\right)=\gamma I\left(\xi_{B}+\xi_{C}\right)+(\beta-\gamma) I\left(\xi_{B}\right),
$$

and similarly, for $\gamma \geq \beta$. One can show $I$ is an integral and $\xi_{A}, \xi_{B}+\xi_{C}, \xi_{A}$ $+\xi_{B}+\xi_{C}$ are measurable. Hence if $D \subset X$ is $\mu$-measurable then $\xi_{D}$ is measur- 
able. However, $\mu$ is not a regular outer measure since $\mu(B) \neq \mu(B \cup C)$. If it were true that $\mu(B)=\mu(C)=\mu(B \cup C), \mu$ would be a regular outer measure.

Even though the 'equivalency' of $A$ and $\xi_{A}$ as measurable entities is not limited to regular outer measures an example may indicate what can happen if $\mu$ is not a regular outer measure. Let $C, D$ be the same sets as before Definition 1.1. (An analogous discussion can be given using the sets $B, C$ of the example just above.) Lebesgue measure $\lambda$ as defined in $[2,9.19]$ is a regular outer measure and it is true that $\lambda(C)=\lambda(D)=\lambda(C \cup D)>0$ and $C \cup D$ is $\lambda$-measurable. The integral $\mathbf{U}$ is defined such that $\mathbf{U}_{R} \xi_{A} d \lambda=\lambda(A)$. For $\gamma \geq \delta \geq 0$ it is true that

$$
\mathbf{U}_{R} \gamma \xi_{C}+\delta \xi_{D} d \lambda=\mathbf{U}_{R} \gamma \xi_{C} d \lambda=\gamma \lambda(C)
$$

However, if one goes to an outer measure $\mu$ which is identical to $\lambda$ on the $\lambda$-measurable sets so that $\lambda(C \cup D)=\mu(C \cup D)$ the only restriction placed on $\mu$ is that $\mu(C)+\mu(D) \geq \mu(C \cup D)$. For any integral which induces $\mu$ it is true that $I\left(\xi_{C}\right)+I\left(\xi_{D}\right) \geq I\left(\xi_{C}+\xi_{D}\right)=\lambda(C \cup D)$, and, for $\gamma \geq \delta>0$,

$$
\begin{aligned}
\max \left\{I\left(\gamma \xi_{C}\right)\right. & \left., I\left(\delta \xi_{D}\right), \gamma I\left(\xi_{C}+\xi_{D}\right)-(\gamma-\delta) I\left(\xi_{D}\right)\right\} \\
& \leq I\left(\gamma \xi_{C}+\delta \xi_{D}\right) \\
& \leq \min \left\{I\left(\gamma \xi_{C}\right)+I\left(\delta \xi_{D}\right), \delta I\left(\xi_{C}+\xi_{D}\right)+(\gamma-\delta) I\left(\xi_{C}\right)\right\}
\end{aligned}
$$

while

$$
\max \left\{\mathbf{U}_{R} \gamma \xi_{C} d \lambda, \mathbf{U}_{R} \delta \xi_{D} d \lambda\right\}=\mathbf{U}_{R} \gamma \xi_{C}+\delta \xi_{D} d \lambda<\mathbf{U}_{R} \gamma \xi_{C} d \lambda+\mathbf{U}_{R} \delta \xi_{D} d \lambda .
$$

Thus even once $\mu$ has been determined $I$ can take on any value allowed by the inequality. The value of $\mathbf{U}$, however, is uniquely determined by its regular outer measure $\mu$. Hence if $\mu$ is a regular outer measure the value of $I$ is known; if $\mu$ is not a regular outer measure the value of $I$, in the absence of more complete information, may only be known to be bounded by a set of inequalities. However, knowing no more than $I$ is an integral the following theorem can still be obtained.

Theorem 3.12. Let I be an integral on a set $X$. Let $f$ be a nonnegative function such that $I(f)$ is finite. For $\epsilon>0$ there exist sequences $\left(\alpha_{n}\right)_{n=1}^{\infty}$, $\left(\beta_{n}\right)_{n=1}^{\infty}$ such that for all $n \in N, 0 \leq \alpha_{n} \leq \beta_{n} \leq \infty$, and a sequence of subsets of $X,\left(A_{n}\right)_{n=1}^{\infty}$, such that

and

$$
\sum_{n=1}^{\infty} \alpha_{n} \xi_{A_{n}} \leq f \leq \sum_{n=1}^{\infty} \beta_{n} \xi_{A_{n}}
$$




$$
\begin{aligned}
& \epsilon>I\left(\sum_{n=1}^{\infty} \beta_{n} \xi_{A_{n}}-\sum_{n=1}^{\infty} \alpha_{n} \xi_{A_{n}}\right) \geq I\left(\sum_{n=1}^{\infty} \beta_{n} \xi_{A_{n}}\right)-I\left(\sum_{n=1}^{\infty} \alpha_{n} \xi_{A_{n}}\right), \\
& \epsilon>I\left(f-\sum_{n=1}^{\infty} \alpha_{n} \xi_{A_{n}}\right) \geq I(f)-I\left(\sum_{n=1}^{\infty} \alpha_{n} \xi_{A_{n}}\right), \\
& \epsilon>I\left(\sum_{n=1}^{\infty} \beta_{n} \xi_{A_{n}}-f\right) \geq I\left(\sum_{n=1}^{\infty} \beta_{n} \xi_{A_{n}}\right)-I(f) .
\end{aligned}
$$

If $I(f)>0$ the $\beta_{n}$ can be chosen such that

$$
\alpha_{n} \leq \beta_{n} \leq(1+\epsilon / I(f)) \alpha_{n} .
$$

Proof. Let $\beta>0$. Define for all integer $n A_{n}=\left\{x: x \in X,(1+\beta)^{n} \leq f(x)<\right.$ $\left.(1+\beta)^{n+1}\right\}$. Let

$$
A_{\infty}=\{x: x \in X, f(x)=\infty\}, \quad A_{-\infty}=\{x: x \in X, f(x)=0\} .
$$

Define $g=\sum_{n=-\infty}^{\infty}(1+\beta)^{n} \xi_{A_{n}}$. It is true that $g \leq f$ and $I(g) \leq I(f)$. However $f \leq(1+\beta) g$ and $I(f) \leq(1+\beta) I(g)$. Choose $\beta$ such that $\beta I(f)<\epsilon$. It is true that $I(f-g) \leq I((1+\beta) g-g)=\beta I(g) \leq \beta I(f)<\epsilon$. The theorem follows by setting $\alpha_{n}=(1+\beta)^{n}, \beta_{n}=(1+\beta) \alpha_{n}$, reordering the indexing, and noticing that

$$
I(f)=I(f-g+g) \leq I(f-g)+I(g)
$$

so

$$
I(f)-I(g) \leq I(f-g)<\epsilon .
$$

\section{BIBLIOGRAPHY} 504 .

1. P. R. Halmos, Measure theory, Van Nostrand, Princeton, N. J., 1950. MR 11,

2 E. Hewitt and K. Stromberg, Real and abstract analysis. A modern treatment of the theory of functions of a real variable, Springer-Verlag, New York, 1965. MR 32 \#5826. 02138

DEPARTMENT OF CHEMISTRY, HARVARD UNIVERSITY, CAMBRIDGE, MASSACHUSETTS

Current address: Department of Chemistry, University of California at San Diego, La Jolla, California 92037 